\title{
DEVELOPMENT OF A CRYOGENIC RADIATION DETECTOR FOR MAPPING RADIO FREQUENCY SUPERCONDUCTING CAVITY FIELD EMISSIONS
}

\author{
D. Dotson, John Mammosser, Jefferson Lab, Newport News, VA. 23606, USA
}

\section{Abstract}

Field emissions in a super conducting helium cooled RF cavity and the production of radiation (mostly XRays) have been measured externally on cryomodules at Jefferson Lab since 1991. External measurements are limited to radiation energies above $100 \mathrm{keV}$ due to shielding of the stainless steel cryogenic body. To measure the onset of and to map field emissions from a superconducting cavity requires the detecting instrument be inside the shield and within the liquid Helium. Two possible measurement systems are undergoing testing at JLab. A CsI detector array set on photodiodes and an XRay film camera with a fixed aperture. Several devices were tested in the cell with liquid Helium without success. The lone survivor, a CsI array, worked but saturated at high power levels due to backscatter. The array was encased in a lead shield with a slit opening set to measure the radiation emitted directly from the cell eliminating a large portion of the backscatter. This is a work in progress and testing should be complete before the PAC 05. The second system being tested is passive. It is a shielded box with an aperture to expose radiation diagnostic film located inside to direct radiation from the cell. Developing a technique for mapping field emissions in cryogenic cells will assist scientists and engineers in pinpointing any surface imperfections for examination.. This work was supported by DOE contract DE-AC0584ER40150

\section{INTRODUCTION}

Measurements of field emissions on production cavities for Jefferson Lab at the RF feeds (top Hats) and beam line produced data indicating at what RF power an internal arc would occur which correlated nicely with the field emission measurements. These were all external measurements of energies above $100 \mathrm{keV}$ due to the cavity wall shielding. Measuring the field emissions of a radio frequency inside the cryogenic enclosure would allow for the early detection and mapping of field emissions on the surface of the modules at a much lower energy. The search was on to find a detector which could stand up to temperature cycling and extremely high radiation fields and be cost effective for a production facility.A number of detector types and materials were tested in liquid nitrogen . One candidate, a CsI array on a photodiode substrate emerged as a strong candidate for a

*Work supported by the US. Department of Energy under contract DE DE-AC05-84ER40150; US patent pending. prototype test. It is an "off the shelf" sixteen detector array $1 \mathrm{~cm}$ by $3 \mathrm{~cm}$ designed to measure X-Rays. The second system is a simple lead box with a hole drilled through to expose dental film packets mounted inside. A shutter system operating from a gear shaft is under design to shield the film after exposure.

\section{THE DETECTOR}

The CsI detector chosen for a read world test in a module. The basic unit has sixteen individual detectors set on a photosensitive substrate. It was placed in an aluminum box and connecting wires run through feed through connectors to the readout. Several cryogenic cycles were completed successfully with no damage to the detector. A problem encountered was the intensity of the radiation field caused the unshielded detector to saturate at higher cavity gradients due to backscatter and all sixteen detectors were wired to act as one.

\section{SHIELDING THE DETECTOR}

A lead shield with an aperture slit set to measure the field emissions from one of the seven cells of a superconducting module was designed and constructed (this is illustrated by Figure 1A and 1B). The proposed production model will have a detector for each of the seven cells). The detector was set on end facing the radiation source and the detectors were wired in groups of four. The first four detectors are closest to the aperture and the last four (this is illustrated by Figure 2) at the rear of the shield. This arrangement could provide a crude energy measurement as well as an overall field strength. Preliminary measurements on the bench indicate that the higher the energy the further the penetration of the crystals.

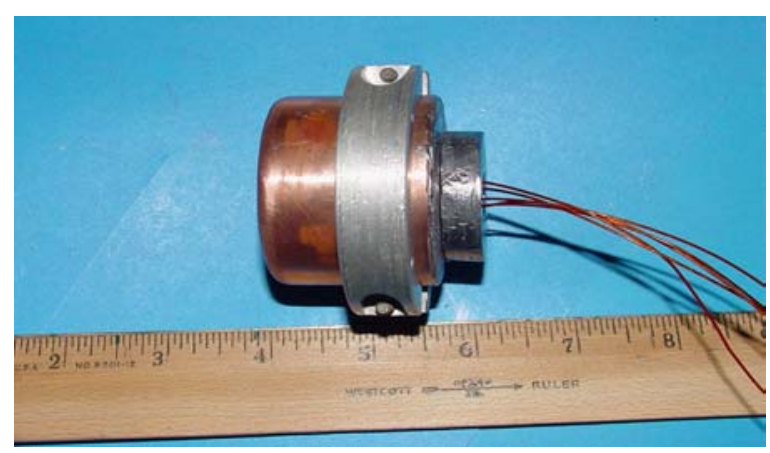

Figure 1A: Assembled Detector 


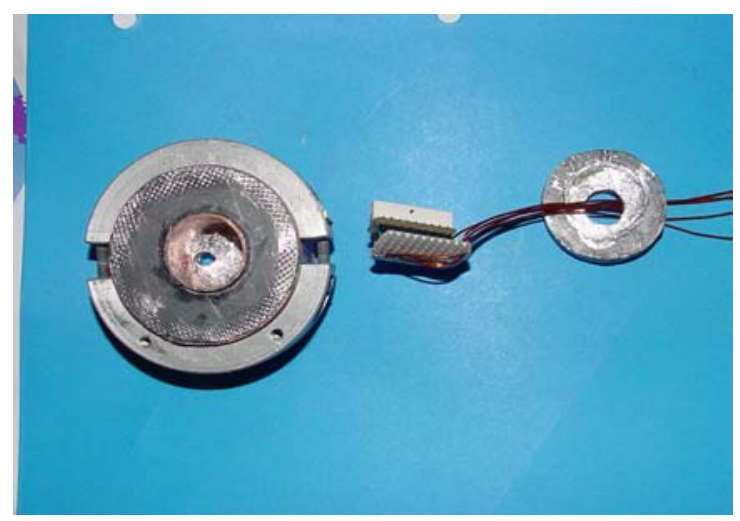

Figure 1B: Assembled Detector

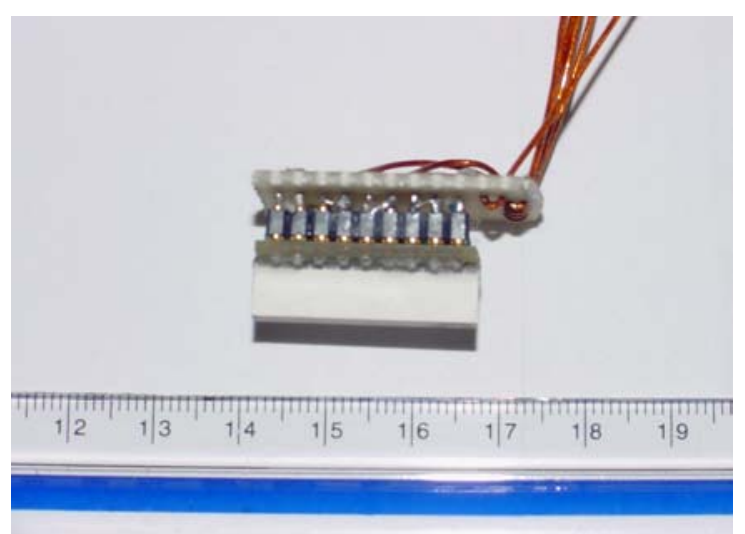

Figure 2: CsI Detector

\section{WORK IN PROGRESS}

Seven detectors will be mounted on an existing arm inside the testing vessel which travels 360 degrees around the cells under test (this is illustrated by Figure 4). The output from the detectors will be sent to an ADC which is in sync with the rotation around the cells radiation from the surface of each cell will be mapped for surface emissions and energy. Studying the use of the CsI detectors in a feed back loop to turn power down on a particular module to allow dissipation of the window field emissions prior to an arc trip and up on another which has "head room". When the field emissions fall to a pre determined level (about 10\%) the power will be restored to the cavity and the overall power output of the accelerator will not change through the cycle. This will hopefully lower the arc rate decreasing the down time.

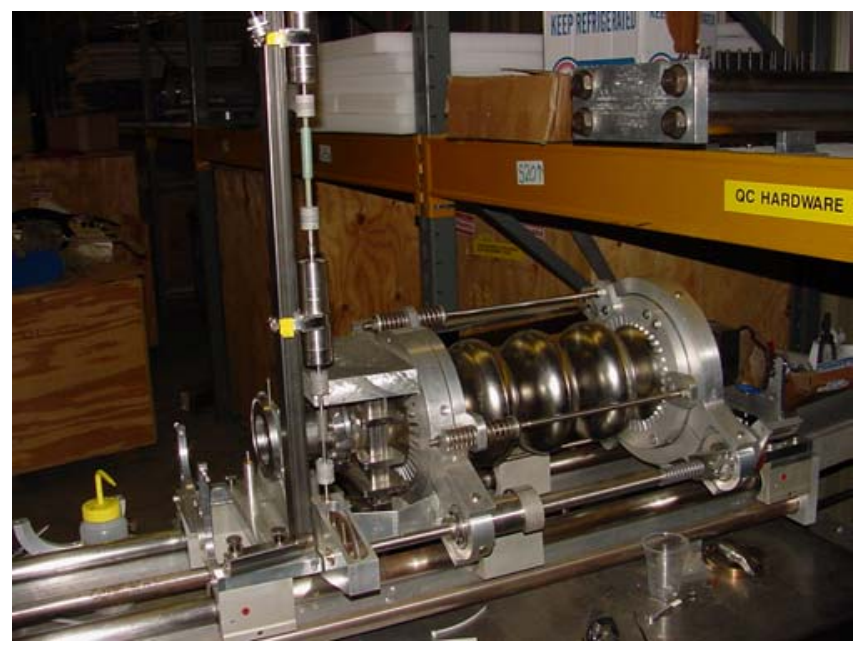

Figure 4: Cryo Cell

ental film is placed inside a shield box with an aperture which will produce a negative "picture" of the XRay emissions from the surface of the cells. The shutter (a lead plug) will be activated from outside the test vessel at a predetermined exposure as measured by the CsI detector.

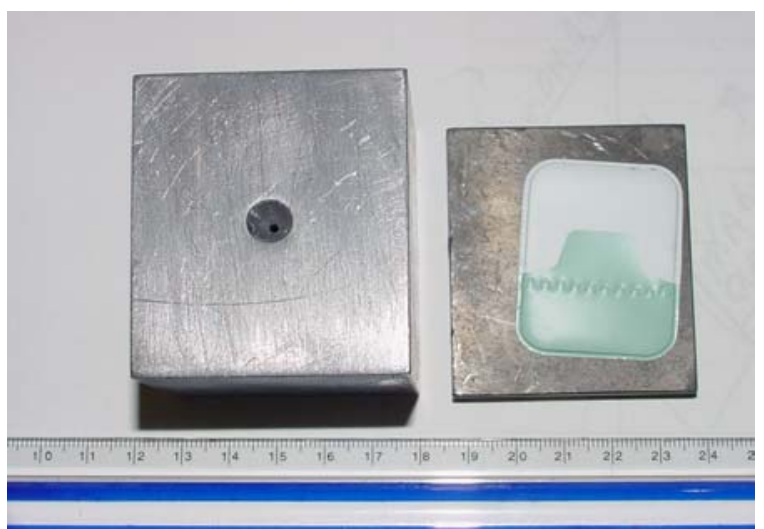

Figure 3: X-ray Camera 